University of Texas at El Paso

ScholarWorks@UTEP

$6-2020$

\title{
Healthy Lifestyle Decreases the Risk of Alzheimer Disease: A Possible Partial Explanation of an Empirical Dependence
}

Olga Kosheleva

The University of Texas at El Paso, olgak@utep.edu

Vladik Kreinovich

The University of Texas at El Paso, vladik@utep.edu

Follow this and additional works at: https://scholarworks.utep.edu/cs_techrep

Part of the Applied Mathematics Commons

Comments:

Technical Report: UTEP-CS-20-63

\section{Recommended Citation}

Kosheleva, Olga and Kreinovich, Vladik, "Healthy Lifestyle Decreases the Risk of Alzheimer Disease: A Possible Partial Explanation of an Empirical Dependence" (2020). Departmental Technical Reports (CS). 1475.

https://scholarworks.utep.edu/cs_techrep/1475

This Article is brought to you for free and open access by the Computer Science at ScholarWorks@UTEP. It has been accepted for inclusion in Departmental Technical Reports (CS) by an authorized administrator of ScholarWorks@UTEP.For more information, please contact Iweber@utep.edu. 


\title{
Healthy Lifestyle Decreases the Risk of Alzheimer Disease: A Possible Partial Explanation of an Empirical Dependence
}

\author{
Olga Kosheleva and Vladik Kreinovich \\ University of Texas at El Paso \\ $500 \mathrm{~W}$. University \\ El Paso, TX 79968, USA \\ olgak@utep.edu, vladik@utep.edu
}

\begin{abstract}
A recent paper showed that for people who follow all five healthy lifestyle recommendations, the risk of Alzheimer disease is only $40 \%$ of the risk for those who do not follow any of these recommendations, and that for people two or three of these recommendations, the risk is $63 \%$ of the not-followers risk. In this paper, we show that a relation between the two numbers - namely, that 0.40 is the square of 0.63 - can be naturally explained by a simple model.
\end{abstract}

\section{Formulation of the Problem}

A recently discovered empirical dependence. A recent paper [1] analyzed the effect of the following five healthy lifestyle factors on the risk of Alzheimer disease:

- non-smoking,

- physical activity - at least 150 minutes per week of moderate-to-vigorous intensity physical activity,

- light to moderate alcohol consumption,

- heathy diet - as measured by belonging to the upper $40 \%$ in terms of healthy eating, and

- engagement in late-life cognitive activities - also as measured by belonging to the upper $40 \%$ in terms of such activities.

The main conclusion is that these factors drastically decrease the risk of Alzhemier disease in comparison with participants who do not have any of these factors. Specifically: 
- for participants that had all five healthy lifestyle factors, the risk was $40 \%$ of the risk of no-factors folks - i.e., the proportion of fully-healthy-lifestyle folks who got this disease was 2.5 time smaller than among those who did not have a healthy lifestyle at all; and

- for participants that had two to three healthy lifestyle factors, the risk was $63 \%$ of the risk of those who did not have a healthy lifestyle at all.

How can we explain this empirical dependence? A natural question is how can we explain these empirical results.

What we do in this paper. In this paper, we provide a possible partial explanation of these results. Namely, while we cannot explain neither of the two empirical percentages, we use simple ideas to explain the relation between the two risk reduction numbers.

\section{Possible Explanation}

Towards a simple model: first idea. Most probably, each of the five factors $i$ decreases the risk of the Alzheimer disease: i.e., as a result of this factor, the proportion of people with this factor who get this disease is smaller by some multiplicative constant $c_{i}$ that the proportion of Alzheimer patients among people who do not have a single factor.

We do not know how exactly each of the five factors affects the risk of getting Alzheimer disease. Thus, we have no reason to assign different value $c_{i}$ to different factors. In such situations, a natural idea is to assign the same value to all the factors. This idea - going back to ancient times - was first explicitly formulated by Laplace and is, thus, known the Laplace Indeterminacy Principle; see, e.g., [2]. Not only it makes sense, in its modern general form of Maximum Entropy Method this principle has led to many successful data processing techniques [2].

This principle is mostly applied to probabilities - and it is when applied to probabilities, this principle has been most empirically successful. Good news is that in our case, the factors $c_{i}$ can also be interpreted as probabilities: namely, as a probability that a person who would have gotten the Alzheimer disease if he or she did not lead a healthy lifestyle will still get this disease if he/she follows the $i$-th factor.

By applying Laplace Indeterminacy Principle, we select a model in which for all five factors listed above, this probability is the same. We will denote this common probability by $c$, i.e., we have $c_{1}=\ldots=c_{5}=c$.

Towards a simple model: second idea. What if a person has several factors? Fir example, we know the probability $c_{1}$ that a person will still have this bad luck (i.e., Alzheimer disease) in the presence of the first factor, and we know the probability $c_{2}$ that a person will still have this disease in the presence of the second factor. What is the probability that this person get the disease if he/she 
has both factors - i.e., what is the probability that this person will have both bad lucks?

It is well known that the probability of two events both occurring is not uniquely determined by the probability of each event: the results may be different depending on how related are these events. In our case, we do not have any information about the relation between different factors. So, a reasonable idea is again to use the Laplace Indeterminacy Principle - or, to be more precise, the Maximum Entropy approach. According to this approach, if we know the probabilities of two events, and we have no information about the relation between these events, we assume that these events are statistically independent - i.e., that the probability of these two events both occurring is equal to the product of the probabilities.

Resulting model. In our case, this means that if a person has two factors, his/her risk is equal to $c \cdot c=c^{2}$ of the risk of those who have no factors. Similarly, if a person has $f$ factors, then this person's risk is equal to $c^{f}$ of the risk of those who have no factors.

This simple model indeed explains the relation between empirical values. From the empirical study, we know two numbers:

- the risk decrease of 0.40 corresponding to the case when all five factors are present, and

- the risk decrease of 0.63 corresponding to the case when only two to three factors are present.

Let us compare these numbers with the predictions of our simple model.

For the case of all five factors, according to our model, the corresponding decrease in risk is equal to $c^{5}$. So, to match our model with the empirical data, we must have $c^{5} \approx 0.40$.

To describe the case of 2 or 3 factors, it makes sense to use the intermediate value $f=2.5$, and estimate the resulting decrease in risk as $c^{2.5}$. So, comparing this estimate with the empirical data, we should have $c^{2.5} \approx 0.63$.

How are these two estimates for a parameter $c$ compatible? Actually, they are perfectly compatible: It is easy to see that, in general, $c^{5}=\left(c^{2.5}\right)^{2}$. And in our case, $0,63^{2}=0.3979$, i.e., with the 2 -digit accuracy, $0.63^{2}$ is exactly $0.40-$ so we have a perfect match with our simple model.

Let us summarize what exactly we explained and what we did not explain. We did not explain the value 0.40 , and we did not explain the value 0.63 , but we did explain the fact that the square of the second value is exactly equal to the first value!

What other conclusions can we make from our simple model. From $c^{5} \approx 0.40$, we conclude that $c \approx 0.83$. Thus, we can conclude the following:

- that for participants with one healthy lifestyle factor, the percent of Alzheimer diseases will be approximately $83 \%$ of the percentage among those who do not have any factors; 
- that for participants with two healthy lifestyle factors, the percent of Alzheimer diseases will be approximately $69 \%$ of the percentage among those who do not have any factors;

- that for participants with three healthy lifestyle factors, the percent of Alzheimer diseases will be approximately $57 \%$ of the percentage among those who do not have any factors;

- that for participants with four healthy lifestyle factors, the percent of Alzheimer diseases will be approximately $47 \%$ of the percentage among those who do not have any factors.

\section{Acknowledgments}

This work was supported in part by the National Science Foundation grants 1623190 (A Model of Change for Preparing a New Generation for Professional Practice in Computer Science) and HRD-1242122 (Cyber-ShARE Center of Excellence).

\section{References}

[1] K. Dhana, D. A. Evans, K. B. Rajan, D. A. Bennett, and M. C. Morris, "Healthy lifestyle and the risk of Alzheimer dementia: Findings from 2 longitudinal studies", Neurology, 2020, June 2020

10.1212/WNL.000000000009816; DOI: 10.1212/WNL.0000000000009816.

[2] E. T. Jaynes and G. L. Bretthorst, Probability Theory: The Logic of Science, Cambridge University Press, Cambridge, UK, 2003. 